\title{
Endogenous c-Jun N-terminal kinase (JNK) activity marks the boundary between normal and malignant granulosa cells
}

Gamze Bildik', Nazli Akin', Filiz Senbabaoglu', Yashar Esmalian', Gizem Nur Sahin', Defne Urman', Sercin Karahuseyinoglu², Umit Ince ${ }^{3}$, Erhan Palaoglu ${ }^{4}$, Cagatay Taskiran ${ }^{5}$, Macit Arvas ${ }^{6}$, Yilmaz Guzel ${ }^{6}$, Kayhan Yakin ${ }^{7}$ and Ozgur Oktem ${ }^{7}$

\section{Abstract}

Granulosa cell tumor of the ovary (GCT) is a very rare tumor, accounting for only $2 \%$ of all ovarian tumors. It originates from sex cords in the ovary and can be divided into adult (95\%) and juvenile (5\%) types based on histologic findings. To date, no clear etiologic process has been identified other than a missense point mutation in the FOXL2 gene. Our previous works showed that c-Jun N-terminal kinase (JNK) pathway plays critical role in cell cycle progression and mitosis of normal and immortalized granulosa cells and follicle growth in rodent ovaries. These findings led us to investigate the role of JNK pathway in the granulosa cell tumor of the ovary. We used two different GCT cell lines (COV434 and KGN) and fresh GCT samples of adult and juvenile types obtained from the patients during surgery. We have discovered that endogenous kinase activity of JNK is markedly enhanced in the GCT samples and cell lines, whereas it was almost undetectable in mitotic non-malignant human granulosa cells. The inhibition of JNK pathway in GCT cell lines with two different pharmacologic inhibitors (SP600125 and AS601245) or siRNA resulted in a dosedependent reduction in in vitro cell growth, increased apoptosis and diminished estradiol and AMH productions. JNK inhibition was also associated with a decrease in the number of cells positive for mitosis marker phospho-histone $\mathrm{H}^{\text {Ser }}$

10 in the asynchronous cells; and diminished EdU uptake during S phase and cell cycle arrest at G2/M-phase transition in the synchronized cells. Ex vivo treatment of patient-derived GCT samples with JNK inhibitors for $24 \mathrm{~h}$ significantly decreased their in vitro growth and estradiol and AMH productions. Furthermore, in human GCT xenograft model, in vivo tumor growth was significantly reduced and plasma AMH levels were significantly decreased in SCID mice after administration of JNK inhibitors and siRNA. These findings suggest that targeting JNK pathway may provide therapeutic benefit in the treatment of granulosa cell tumors for which currently no curative therapy exists beyond surgery.
\end{abstract}

\section{Introduction}

Granulosa cell tumor of the ovary (GCT) is a very rare tumor characterized by its tendency to recur years after

\footnotetext{
Correspondence: Ozgur Oktem (ooktem@ku.edu.tr)

${ }^{1}$ Graduate School of Health Sciences and School of Medicines, Koc University, Istanbul, Turkey

${ }^{2}$ Department of Histology and Embryology, School of Medicine, Koc University, Istanbul, Turkey

Full list of author information is available at the end of the article Edited by R. Johnstone
}

the initial diagnosis. It accounts for approximately $2 \%$ of all ovarian tumors and can be divided into adult (95\%) and juvenile $(5 \%)$ types based on histologic findings ${ }^{1,2}$. To date, no clear etiologic process has been identified other than a somatic missense point mutation $(\mathrm{C} 402 \rightarrow \mathrm{G}$; C134W) in the FOXL2 gene that is positive in $97 \%$ of adult-type granulosa cell tumor and absent in its juvenile form $^{3}$. Indeed, recent studies have revealed many genes and signaling pathways that are merged to FOXL2 and 
work as critical regulators of granulosa cell proliferation and function such transforming growth factor- $\beta$ (TGF- $\beta$ ) signaling (GDF-9, follistatin, Smad3), GATA4 and aromatase $^{4-6}$. Unlike the adult type, juvenile-type GCT (JGCT) is much rarer, does not harbor FOXL2 mutations and affects pre-pubertal girls and young women with a mean age of onset of around 8 years ${ }^{7,8}$. Its molecular mechanism is less known compared to adult type. One study detected in-frame tandem duplications within AKT1 as well as an array of point mutations altering highly conserved residues in a cohort of 16 JGCTs $^{9}$. JGCTs exhibit reduced expression of FOXL2 compared to normal ovary ${ }^{10}$. Pre-ovulatory growth of the somatic cells of the ovary is induced by the follicle-stimulating hormone (FSH), and alterations in its signaling pathway have been suggested to play a role in tumorigenesis. Consistently, two activating mutations of the stimulatory $\alpha$-subunit of a trimeric G protein (Gos), located at position 201 , have been identified in $30 \%$ of a JGCT cohort $^{11}$. The majority of patients diagnosed with adult or juvenile GCT present with an early-stage disease, with a tumor limited to the ovary and have a good prognosis with a survival rate of $>90 \%$ with surgery alone. However, patients with advanced-stage disease and widely spread tumors or recurrent cases have a very poor prognosis and are more difficult to treat. Anti-mullerian hormone (AMH) and estrogen are produced by hormonally active tumors and used as adjuvant hormone markers in the diagnosis and post-treatment follow-up of the patients. Because JGCTs are hormonally active, patients can be diagnosed with precocious pseudopuberty owing to increased estrogen secretion. Indeed, there are no other curative treatment forms other than surgery ${ }^{9,12,13}$.

Mitogen-activated protein kinases (MAPKs) are the members of a well-studied family of serine-threonine kinases that phosphorylate target proteins and play important regulatory roles in the cell. ${ }^{14}$ The c-Jun NH2terminal kinases (JNKs), a member of MAPKs, are the master protein kinases that regulate many physiological processes, including inflammatory responses, cell proliferation, differentiation, survival and death ${ }^{15,16}$. Our previous work showed that FSH activates JNK pathway in rat granulosa cells, and when this pathway was blocked by pharmacological inhibitors, in vitro follicle growth is halted as a result of mitotic arrest in the granulosa cells surrounding the oocyte in the mouse model. We also found that JNK inhibition in spontaneously immortalized rat granulosa cells (SIGC) resulted in cell cycle arrest at $\mathrm{G}_{2} / \mathrm{M}$ transition ${ }^{17,18}$. This cell line represents an intermediate step in carcinogenesis because they grow indefinitely in culture but do not form clones in soft agar or tumors in nude mice ${ }^{19}$. Based on these findings we hypothesized that JNK pathway might have a role in GCT. For this purpose, we utilized fresh tumor samples of adult and juvenile types as well as two different GCT cell lines (COV434 and KGN) in comparison to immortalized mitotic non-luteinizing FSH-responsive human ( $\mathrm{HGrC1}$ ) and rat (SIGC) granulosa cell lines and primary human luteinized granulosa cells (HLGCs) obtained from In vitro fertilization (IVF) patients in this translational research study. Two different pharmacological JNK inhibitors (SP600125 and AS601245) and small interfering RNA (siRNA) technology were used to explore the role of JNK pathway in human GCT.

\section{Results \\ Validation experiments}

First, we conducted a series of validation experiments to test if our experimental model is suitable to study the role of JNK pathway in GCT cell lines and tumor samples. We previously determined in vitro growth characteristics of GCT cell lines COV434 and KGN, and mitotic nonmalignant human ( $\mathrm{HGrC1}$ ) and rat (SIGC) granulosa cells $^{20}$. In GCT cell line COV434, FSH stimulation at different concentrations increased the expression of phospho-c-Jun $^{\text {Ser63 }}$ in a dose-dependent fashion. Treatment with JNK inhibitors at $12.5,25,50$ and $100 \mu \mathrm{mol} / \mathrm{L}$ concentrations $30 \mathrm{~min}$ before FSH abolished the expression of phospho-c-Jun ${ }^{\mathrm{Ser63}}$ in a dose-dependent manner on western blotting (Fig. 1a) and immunofluorescence staining at low- and high-magnification images (Fig. 1b). Similarly, knockdown of JNK by siRNA at 25, 50 and 100 $\mathrm{nM}$ concentrations caused a dose-dependent decrease in the expression of protein level of total JNK (Fig. 1c). Inhibition of JNK pathway after treatment with pharmacological inhibitors and siRNA at the indicated concentrations was confirmed by measuring JNK activity with phosphorylation of c-Jun at Ser63 and 73 residues in GCT cell lines COV434 and KGN (Fig. 1d and supplementary figure-1).

\section{Inhibition of JNK pathway blocks proliferation and cell cycle progression of GCT cell lines}

The interruption of JNK pathway via either pharmacologic inhibitors (SP600125 and AS601245) or siRNA at the indicated concentrations resulted in a dose-dependent decrease or arrest in the proliferation of both COV434 and KGN cells as evidenced by real-time and quantitative assessment of growth curves of the cells and their mean cell indices in the xCELLigence system (Fig. 2 and supplementary figure-2). In line with these results, in vitro estradiol and AMH production of the cells were significantly decreased when JNK pathway was inhibited either pharmacologically or via siRNA (Fig. 3).

In asynchronous COV434 cells, reduced growth was also associated with a significant decrease in the number of cells stained positive for mitosis marker phosphohistone $\mathrm{H} 3{ }^{\mathrm{Ser} 10}$ after JNK inhibition with pharmacological 


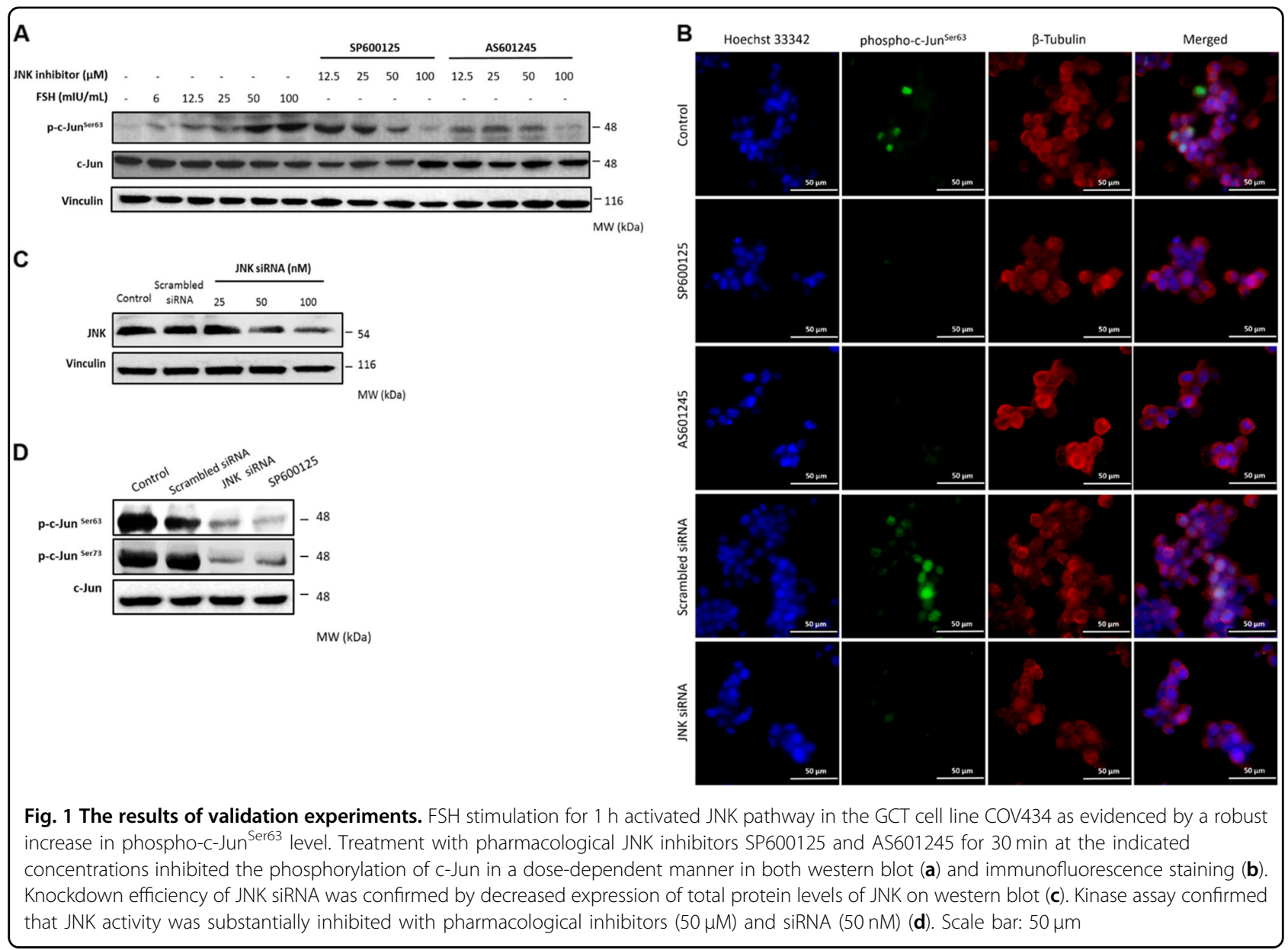

inhibitors (2\%) and siRNA (3\%) compared to control cells $(17 \%, p<0.01)$ on immunofluorescence staining (Fig. 4a, b). A similar reduction in the percentage of the cells positive for phospho-histone $\mathrm{H} 3{ }^{\mathrm{Ser} 10}$ was also observed in asynchronous KGN cell line after JNK inhibition with pharmacological inhibitors and siRNA (Supplementary Figure-3B and 3B).

We further explored the role of JNK pathway in GCT by analyzing cell cycle progression at G1/S and G2/M transitions after JNK inhibition in GCT cell lines. The 5ethynyl-2'-deoxyuridine (EdU) uptake of the COV434 cells synchronized at G1/S was significantly decreased after JNK inhibition with pharmacological inhibitors (6\%) or siRNA (9\%) in comparison to controls $(47 \%, p<0.01$, Fig. 5a, b). Similar results were obtained in KGN cell line (Supplementary Figure-4). JNK inhibition at G2/M transition resulted in failure of the cells to exit mitosis and caused their accumulation at G2/M as evidenced by flow cytometric analysis and absence of de-phosphorylation of p-cdc- $2^{\text {Tyr15 }}$ and degradation of cyclins $A$ and B1 on western blotting (Fig. 5c, d). High-magnification images for the immunofluorescence staining experiments for
phospho-c-Jun and phospho-histone-H3 can be found in the supplementary figure. 5 .

Endogenous kinase activity of JNK is higher in neoplastic granulosa cells compared to primary and immortalized non-neoplastic granulosa cells

Robust anti-proliferative effect of JNK inhibition on the GCT cell lines COV434 and KGN led us to measure JNK activity in GCT cell line and fresh tumor samples in comparison to normal granulosa cells. We found that endogenous kinase activity of JNK is significantly increased in the tumor samples of both adult- and juvenile-type GCT as well as GCT cell lines COV434 and KGN in comparison to other types of non-neoplastic granulosa cells (HGrC1, HLGC and SIGCs) (Fig. 6a, b and Supplementary Figure-6). Ex vivo treatment of fresh GCT samples of both adult and juvenile types with JNK inhibitors in culture resulted in a significant decrease in their in vitro growth and estradiol and $\mathrm{AMH}$ productions compared to control samples (Fig. 6c, d and Supplementary Figure-7). Histological examination and cell death assay with intra-vital fluorescein carbocyanine uptake confirmed decreased viability 


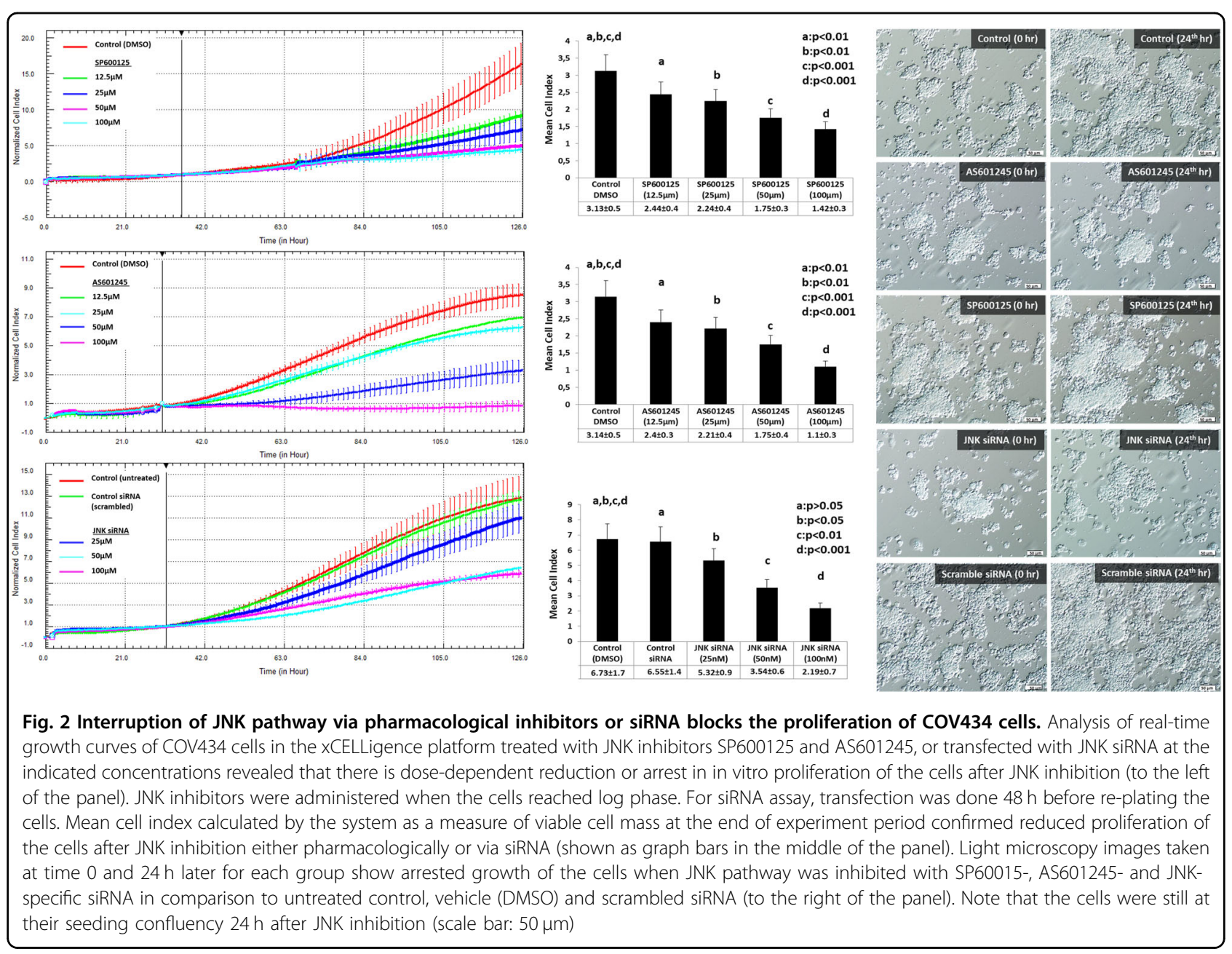

and increased cell death in the samples treated with JNK inhibitors (Fig. 6e, f).

\section{JNK inhibition halts in vivo growth of GCT in mice}

So far, our results showed that the inhibition of JNK pathway via either pharmacologically or RNA interference technology significantly blocked in vitro proliferation of tumor cells, caused cell cycle arrest and decreased in vitro growth of tumor explants. To further substantiate the significance of our findings, we have generated a human GCT xenograft model to analyze the effects of JNK inhibition on in vivo growth of GCT. As shown in the Fig. $7 \mathrm{a}-\mathrm{d}$, in vivo growth of the GCT (COV434) was significantly halted and serum AMH levels were significantly decreased after systemic and intra-tumoral administration of pharmacological JNK inhibitors and JNK siRNA, respectively, in these animals. While control and scrambled siRNA-injected tumors continue to grow in vivo on day 28 post xenografting, such a growth was not observed in those treated with JNK inhibitors or JNK siRNA.

\section{Discussion}

We have obtained several important findings in this study. First, endogenous kinase activity of JNK in GCT samples of adult and juvenile types and cell lines is higher than non-neoplastic granulosa cells. Second, interruption of JNK signaling in GCT cell lines via pharmacologic inhibitors and siRNA inhibited their mitotic proliferation, reduced EdU uptake during $\mathrm{S}$ phase and blocked cell cycle progression at G2/M transition. Third, ex vivo treatment of fresh GCT of tumor samples of both adult and juvenile types with JNK inhibitors was associated with a marked reduction in in vitro growth, hormone productions and viability of these tumors. Fourth, in vivo growth of the GCT cell line (COV434) when xenografted into SCID (severe combined immune deficient) mice was significantly halted and serum AMH levels were significantly decreased after systemic and intra-tumoral administration of pharmacological JNK inhibitors and JNK siRNA, respectively, in these animals. Taken together, these findings suggest that JNK signaling pathway may have a role in the underlying pathogenesis of this rare type of ovarian tumor in humans. 


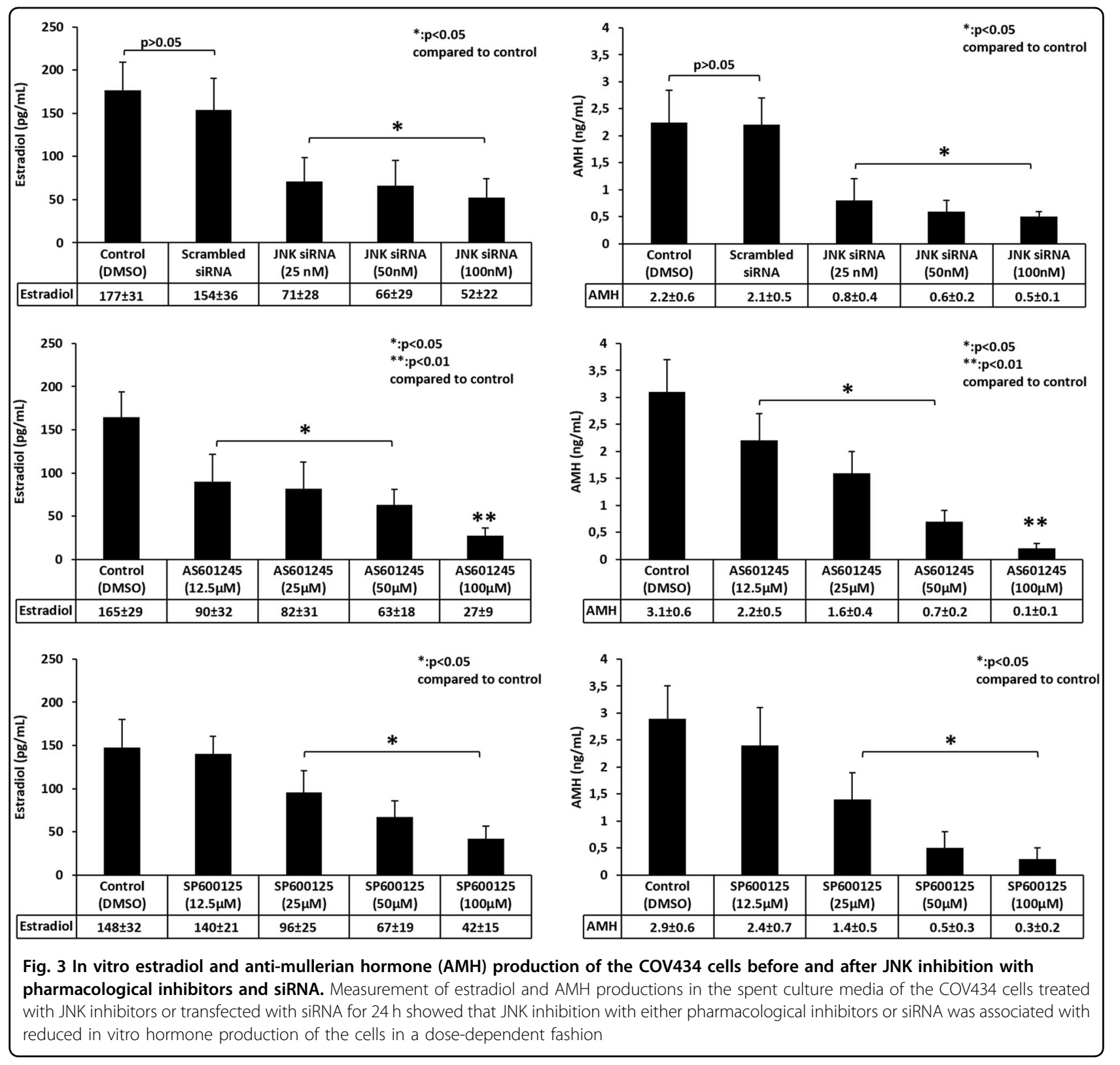

It is well documented that JNK pathway and its downstream effector c-Jun plays crucial role in cell cycle regulation and mitosis of many neoplastic and nonneoplastic cells ${ }^{15}$. Therefore, higher endogenous kinase activity of JNK detected in GCT tumor samples and cell lines might not be surprising. However, absence of such an increase in JNK activity in immortalized mitotically active and rapidly proliferating normal granulosa cells of human ( $\mathrm{HGrC1}$ ) and rat (SIGC) origin suggests that enhanced kinase activity of JNK cannot be solely explained by the presence of mitotic activity in GCT samples and cell lines.

Our study is not the first one that documents the important role of JNK signaling pathway in proliferation and cell cycle progression of malignant tumors as this issue was previously extensively studied in malignant tumors such as the ones showing inhibition of JNK reduces $G 2 / M$ transit independent of $p 53$, leading to endoreduplication, decreased proliferation and apoptosis in the cell lines of breast, lung, thyroid and cervical can$\operatorname{cer}^{21-24}$. However, our findings could be still important given that very limited data are available regarding signaling pathways that act as gate-keeper of the proliferation and apoptosis of granulosa cell tumor. To date, no clear etiologic process has been identified other than a somatic missense point mutation $(\mathrm{C} 402 \rightarrow \mathrm{G} ; \mathrm{C} 134 \mathrm{~W})$ in the FOXL2 gene that is positive in $97 \%$ of adult-type granulosa cell tumor and absent in its juvenile form ${ }^{3}$. 


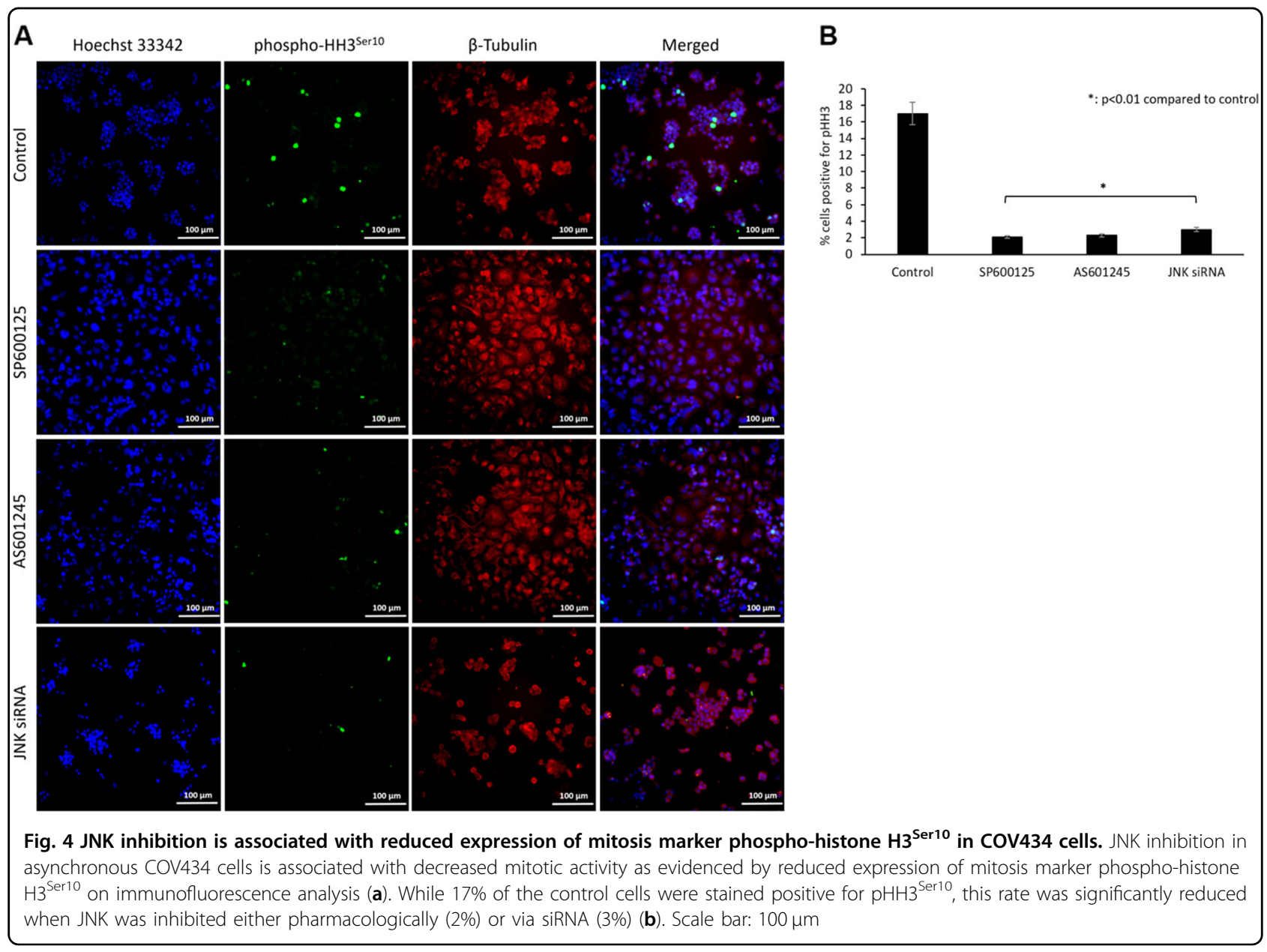

Indeed, recent studies have revealed many genes and signaling pathways that are merged to FOXL2 and work as critical regulators of granulosa cell proliferation and function such as TGF- $\beta$ signaling (GDF-9, follistatin, Smad3), GATA4 and aromatase ${ }^{4-6}$. Unlike the adult type, JGCT is much rarer, does not harbor FOXL2 mutations and affects pre-pubertal girls and young women with a mean age of onset of around 8 years ${ }^{7,8}$. Its molecular mechanism is less known compared to adult type.

Quiescent primordial follicles are composed of a single layer of flattened granulosa cells surrounding the oocyte. Activation of primordial follicles and their transformation into primary stage and beyond is termed initiation of follicle growth and is characterized by squamous to cuboidal transformation of granulosa cells and their further mitotic proliferation. Current evidence suggests that there is a delicate balance of activating and suppressing signaling pathways and the genes that tightly control the process of follicle activation ${ }^{25}$. It is noteworthy that the genes that prevent premature activation of primordial follicles may also cause the formation of granulosa cell tumors when they are mutated. FOXL2 is the first, and probably the most striking, example of how a single gene is a critical regulator of ovarian function, morphology and neoplasia formation in humans. FOXL2 is a winged-helix/ forkhead domain transcription factor. Germ-line knockout of Foxl2 is associated with a failure to assemble primordial follicles ${ }^{26}$, while mutations to Foxl2 cause blepharophimosis/ptosis/epicanthus inversus syndrome (BPES) type I, an autosomal-dominant disorder associated with premature ovarian failure ${ }^{27}$. The relationship between FOXL2 mutations and premature ovarian failure in women with BPES had not been known until 2004, when a transgenic mouse study provided the molecular $\operatorname{link}^{28}$. Five years later, a group of investigators identified a missense point mutation $(402 \mathrm{C}>\mathrm{G}[\mathrm{C} 134 \mathrm{~W}])$ causing a reduction in FOXL2 gene expression in a granulosa cell tumor of the ovary ${ }^{3}$. In the same year, Kobel et al. ${ }^{29}$ identified the same mutation in nearly $90 \%$ of the cases of adult-type granulosa cell tumors. Subsequent studies revealed that the FOXL2 gene is involved in many vital processes of granulosa cells including homeostasis, modulation of the expression of key genes in the differentiation and steroidogenesis, regulation of cell survival 


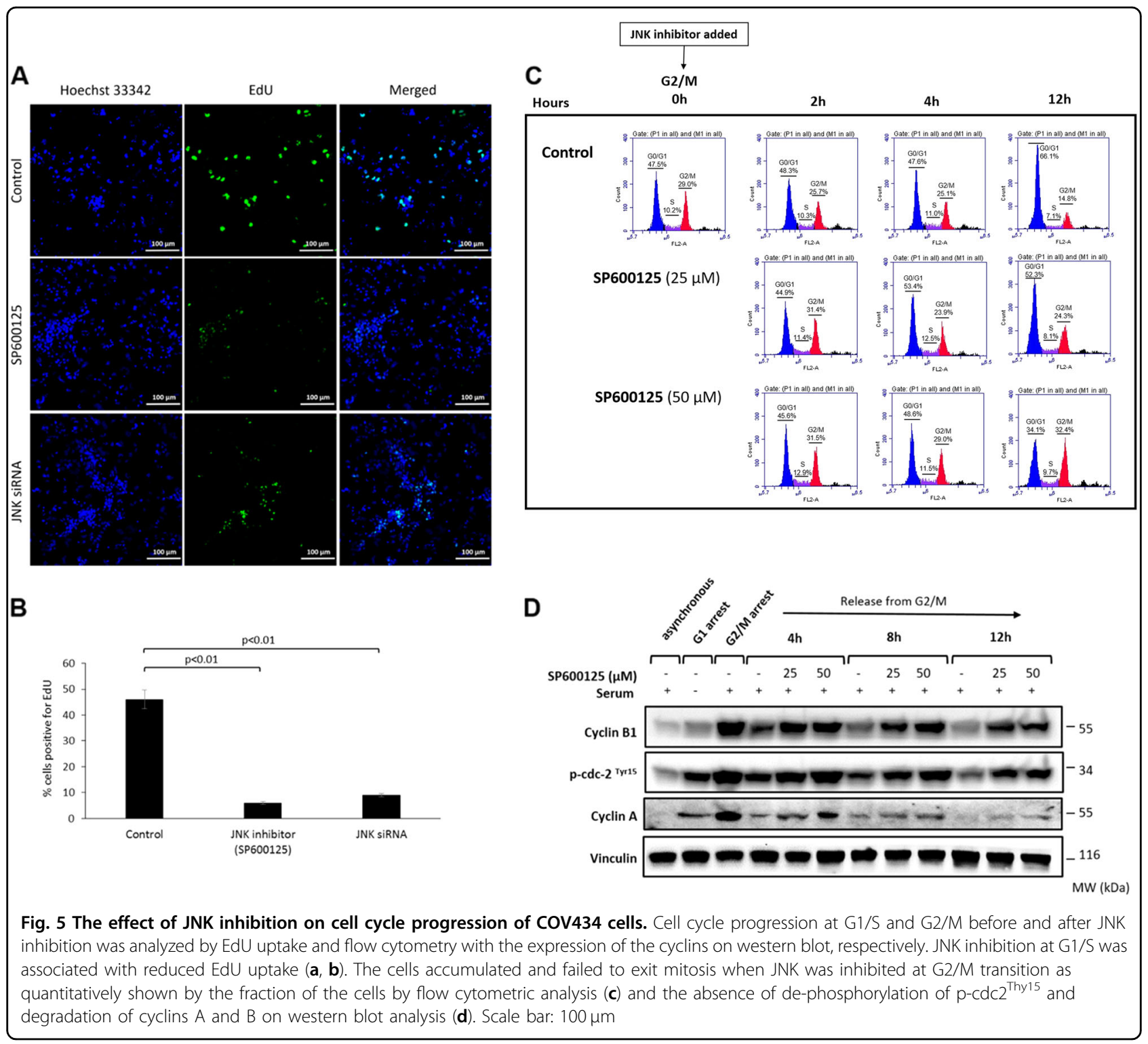

and proliferation and inhibition of cell cycle progression. Furthermore, whereas wild-type FOXL2 can induce apoptotic death of granulosa cells and halt cell cycle progression, the $\mathrm{C} 134 \mathrm{~W}$ mutant cannot, thus providing a survival advantage to malignant granulosa cells ${ }^{6,30-33}$. By the same token, if the FOXL2 gene functions to contribute to follicle dormancy and its mutations can cause premature activation of primordial follicles and/or favor the formation of granulosa cell tumors, can other similar factors or the genes do the same in the granulosa cells? Emerging data from animal studies suggest that Forkhead box O3 (FOXO3), phosphatase and tensin homolog (PTEN) and mammalian target of rapamycin (mTOR) pathways converge on the same follicle dormancy and granulosa cell tumor formation phenotypes. For instance, when Foxo1 and Foxo3 genes were selectively inactivated follicle development is impaired and granulosa cell tumor formation is increased in a mouse model ${ }^{34}$. Moreover, deletion of Pten in this mouse strain enhances the penetrance and accelerates the onset of granulosa cell tumor formation $^{34}$. Other studies showed as well that targeted deletion of Pten alone or in combination with an activating phosphatidylinositol-3-kinase (PI3K) mutation can give rise to granulosa cell tumors in mice ${ }^{35,36}$. However, there is little evidence in humans that links these genes to premature activation of primordial follicles and the formation of granulosa cell tumors. Only two studies in the literature addressed this question at all. The first study by Bittinger et al. ${ }^{37}$ found that the granulosa cell tumor lines, COV434 and KGN, had no mutations within the PTEN gene nor increased expression PIK3CA and PIK3R1, which encode the catalytic and regulatory subunits, 


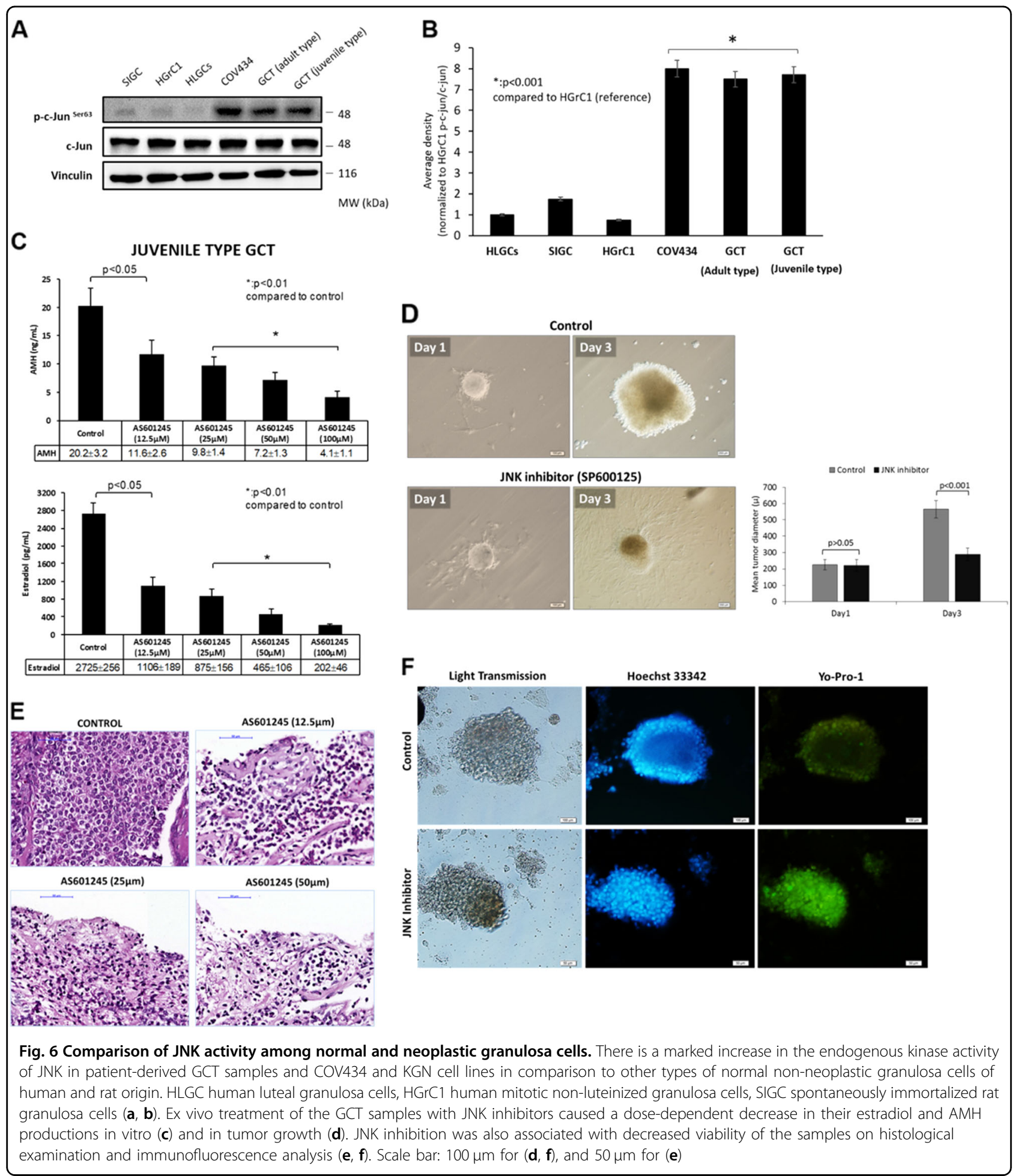

respectively, of PI3K. However, the relevance of these findings to primary human granulosa cell tumor is not clear as the authors could not confirm their results in the surgical GCT samples removed from patients. The second study by Rico et $\mathrm{al}^{38}$ found an increase in the protein abundance of mTOR and its downstream effectorsRPS6KB1 (ribosomal protein S6 kinase B1), RPS6 (ribosomal protein S6), eIF4B (eukaryotic translation initiation factor 4B) and PPARG (peroxisome proliferator-activated receptor gamma) - in three human granulosa cell tumors 

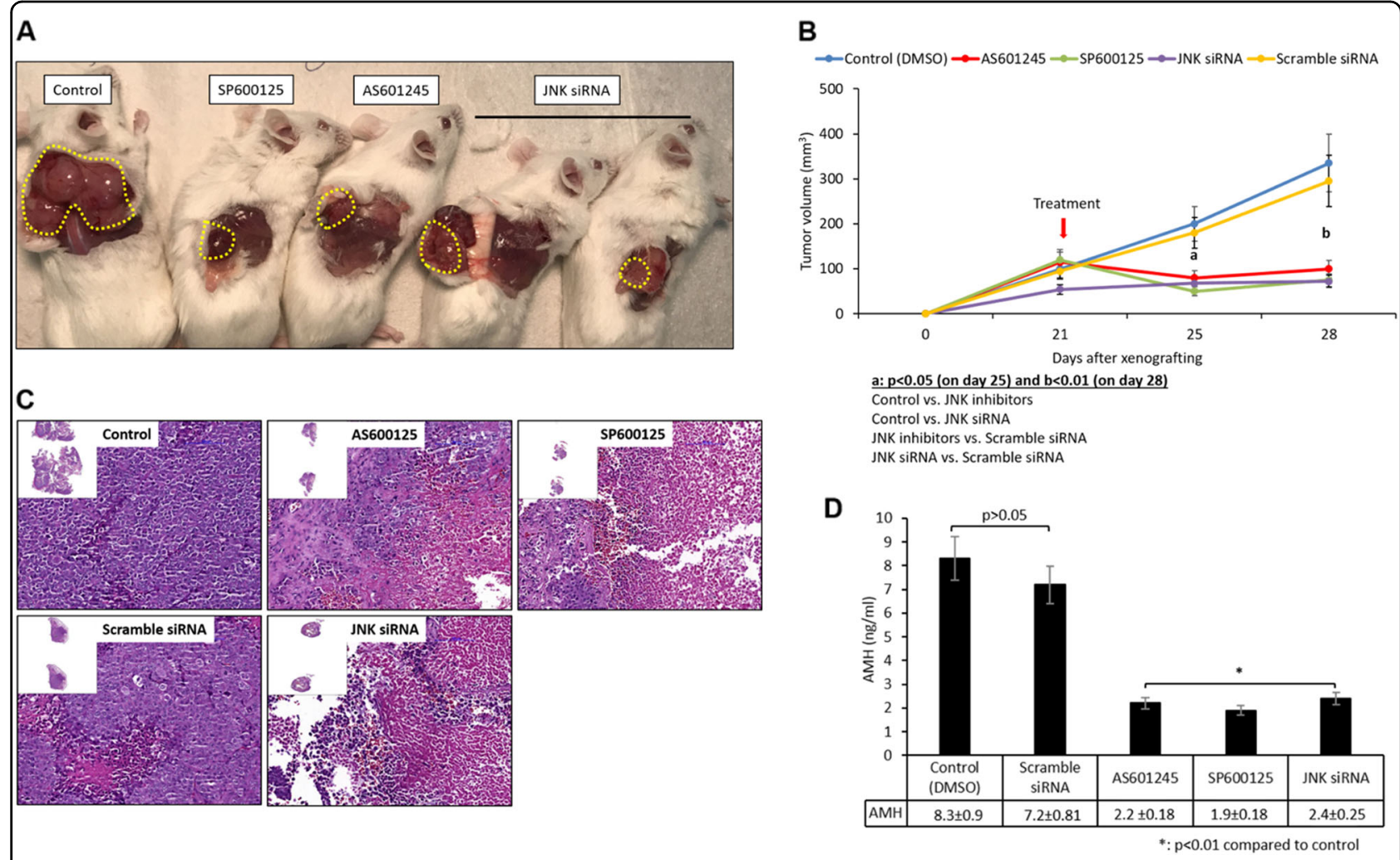

Fig. 7 Human GCT xenograft experiment. Inhibition of JNK pathway either pharmacologically with its specific inhibitors (SP600125 and AS601245) or via siRNA resulted in a significant decrease in in vivo growth of GCT cell line (COV434) when xenografted subcutaneously into SCID mice (a, b). In line with these results, histopathological examination showed that there was a marked decrease in cellularity in the tumors with a concomitant decrease in the serum $\mathrm{AMH}$ level in the animals after JNK inhibitor treatment was given (c, $\mathbf{d})$

samples relative to normal granulosa cells. In the latter study, the investigators also generated a transgenic mouse model in which mTOR, RPS6KB1, eIF4B and PPARG are up-regulated in a manner similar to that found in human granulosa cell tumors. Daily treatment of these animals with the mTOR-specific inhibitor Everolimus for 3 weeks significantly decreased the volume and growth rate of the tumors, and increased the survival of the animals ${ }^{38}$.

\section{Conclusion}

Our findings warrant further investigations since targeting JNK pathway may provide therapeutic benefit in the treatment of granulosa cell tumors for which currently no curative therapy exists beyond surgery.

\section{Materials and methods \\ Patients}

GCT samples of adult $(n=2)$ and juvenile types $(n=2)$ were obtained from 4 patients. Adult and juvenile types of GCT samples were diagnosed and confirmed by two expert pathologists in gynecologic tumors. Non-mitotic HLGCs were isolated from follicular fluid of 10 IVF patients after oocyte retrieval procedure. Informed consents were obtained from all patients and the study was approved by the institutional review board of Koc University.

\section{Chemicals and reagents}

All cell culture materials were obtained from Gibco Inc. SP600125 and AS601245, pharmacological inhibitors of JNK, were purchased from Calbiochem. Control (\#6568) and SAPK/JNK (\#6232) siRNA, SAPK/JNK Kinase Assay Kit (\#8794, nonradioactive), Hoechst 33342 (\#4082), Antic-Jun (\#9165), Anti-Phospho-c-Jun ${ }^{\text {Ser63 }}$ (\#12598), AntiPhospho-c-Jun $^{\text {Ser73 }}$ (\#3270 S), Anti SAPK/JNK (\#9252), Anti-Phospho-SAPK/JNK ${ }^{\text {Thr183/Tyr185 }} \quad(\# 9251), \quad$ AntiCyclin A (4656), Anti-Cyclin B1 (\#12231) and AntiPhospho-cdc-2 ${ }^{\text {Tyr15 }}$ (\#4539) antibodies were obtained from Cell Signaling. All western blotting buffers and reagents were purchased from Bio-Rad. Anti-phosphoHistone $\mathrm{H} 3{ }^{\mathrm{Ser} 10}(06-570)$ antibody was obtained from Upstate. Anti-Vinculin Antibody (V9264) was purchased from Sigma-Aldrich. TransIT-X2 ${ }^{\circledast}$ Dynamic Delivery System (MIR 6000) was purchased from Mirus Bio LLC. YO-PRO $^{\circledR}$-1 Iodide $(491 / 509)$ was obtained from Life Technologies. Texas $\operatorname{Red}^{\mathrm{m}}{ }^{\mathrm{m}}-\mathrm{X}$ Phalloidin and Click-iT ${ }^{\mathrm{mm}}$ 
EdU Alexa Fluor 488 Imaging Kit (C10337) were obtained from Thermo Fisher Scientific. Matrigel Basement Membrane Matrix (356230) was from BD Biosciences. Super Block reagent (\#AAA125) was purchased from ScyTek Laboratories.

\section{Cell lines}

COV434 is a commercially available cell line derived from a granulosa cell tumor. The biological characteristics of this cell line include the production of $17 \beta$-estradiol in response to FSH, the absence of LH receptor, no lutenization capability and the presence of specific molecular markers of apoptosis enabling the induction of follicular atresia $^{39}$.

KGN is also a steroidogenic human ovarian granulosalike tumor cell line derived from a patient with invasive ovarian granulosa cell carcinoma. KGN cells are able to secrete pregnenolone and progesterone with little or no secretion of 17- $\alpha$-hydroxylated steroids, androstenedione or estradiol. Fas-mediated apoptosis was also demonstrated in this cell line, which mimics the physiological regulation of apoptosis in human granulosa cells $^{40}$.

HGrC1 is a human non-luteinized granulosa cell line expressing enzymes related to steroidogenesis, such as steroidogenic acute regulatory protein, aromatase and gonadotropin receptors. These cells are not capable of undergoing luteinization, resembling the characteristics of granulosa cells belonging to follicles in the early stage. HGrC1 might also be capable of displaying the growth transition from a gonadotropin-independent status to gonadotropin-dependent one ${ }^{41}$.

Non-mitotic luteinized human granulosa cells (HLGCs) were recovered from follicular fluid during oocyte retrieval procedure in 10 IVF patients. These cells are highly specialized primary luteinized granulosa cells, and they do not proliferate either spontaneously;or after stimulation with a mitogenic agent. They produce large amounts of progesterone and estradiol hormones in vitro ${ }^{20}$. The aspirates of follicular fluids were spun down at $500 \times g$ for $10 \mathrm{~min}$ and then the cells were recovered and plated.

Spontaneously immortalized rat granulosa cells (SIGC) have an epithelial morphology, express gonadotropin receptors and grow in culture indefinitely. It is a spontaneously immortalized clonal granulosa cell line derived from primary rat ovarian granulosa cells and represents an intermediate step in carcinogenesis because they grow indefinitely in culture but do not form clones in soft agar or tumors in nude mice. ${ }^{19}$ They respond to FSH stimulation with enhanced growth but without undergoing luteinization ${ }^{19}$, resembling the growth characteristics of the proliferating granulosa cells of preantral and early antral follicles as well ${ }^{42,43}$.

\section{Cell culture}

All granulosa cell lines and HLGCs were maintained at $37^{\circ} \mathrm{C}$ with $5 \% \mathrm{CO}_{2}$, in Dulbecco's modified Eagle's medium (DMEM)/F12 supplemented with $10 \%(\mathrm{v} / \mathrm{v})$ fetal bovine serum and $1 \%(\mathrm{v} / \mathrm{v})$ penicillin-streptomycin Amphotericin B Solution, hereafter referred as complete media. The cells were routinely harvested by trypsinization with $0.25 \%$ trypsin-EDTA, counted using a hemocytometer and $0.4 \%$ Trypan blue.

\section{Human GCT tissue culture}

Surgical GCT samples of adult and juvenile types were either freshly lysed for measurement of JNK activity or prepared for in vitro culture. Tumor samples were finely minced and dispersed to cells by treatment with $0.25 \%$ collagenase at $37^{\circ} \mathrm{C}$ for $1 \mathrm{~h}$, plated into 6 -well plates and incubated overnight. The next day, experiment groups were treated with fresh complete media containing JNK inhibitors for $72 \mathrm{~h}$, while the media of control group contained dimethyl sulfoxide (DMSO). Cultured groups were then used for hematoxylin and eosin staining and YO-PRO-1 assay to compare cell viability. Estradiol and AMH levels were measured in culture medium.

\section{Human GCT xenograft model in SCID mice}

GCT cell line COV434 was re-suspended in growth factor reduced Matrigel ${ }^{\circ}$ diluted with DMEM/F12 and xenografted subcutaneously to the right flank region of the SCID mice ( $n=4$ per group) as 1 million cells/300 $\mu \mathrm{l}$ suspension with an insulin syringe. Tumor volume was monitored every week. Once palpable tumor growth was achieved at approximately 3 weeks post transplantation, JNK inhibitors SP600125 (10 mg/kg) and AS601245 (10 $\mathrm{mg} / \mathrm{kg}$ ) were administered systemically in $100 \mu \mathrm{L}$ of DMSO via intraperitoneal route as a single injection (day 21). Control animals received DMSO only. For siRNA experiments, in vitro siRNA transfection experiments were carried out first and then transfected cells were xenografted in the same manner $48 \mathrm{~h}$ later as 1 million cells/300 $\mu$ l suspension with an insulin syringe. JNK or scrambled siRNA were given as a single intra-tumoral injection on day 21. Tumor growth was followed for another week post injection on day 28 , and the animals were killed, tumors were removed and intra-cardiac blood samples were obtained immediately post-mortem for AMH measurement.

\section{SAPK/JNK kinase assay}

Intracellular JNK activity was measured by nonradioactive assay kit as instructed by the manufacturer. Briefly, the cells and tissues were harvested under nondenaturating conditions, lysed on ice and centrifuged at $14,000 \times g$ for $10 \mathrm{~min}$ at $4{ }^{\circ} \mathrm{C}$. Then, $200 \mu \mathrm{l}$ of cell/tissue lysates was incubated with $20 \mu \mathrm{l}$ phospho-JNK rabbit 
monoclonal antibody linked to agarose beads to precipitate JNK enzyme. After addition of necessary buffers, c-Jun substrate and adenosine triphosphate, reaction mixture was incubated for $30 \mathrm{~min}$ at $30{ }^{\circ} \mathrm{C}$, optimal reaction condition that allows c-Jun substrate to be phosphorylated by precipitated JNK. The reaction was stopped by adding $4 \times$ SDS sample buffer and the samples were loaded onto $10 \%$ polyacrylamide gel. Then, proteins were transferred to polyvinylidene difluoride (PVDF) membrane by electroblotting and monoclonal antibodies were used to measure JNK-induced phosphorylation of cJun substrate at Ser63 and Ser73 residues.

\section{Western blotting}

For western blot analysis, the cells were harvested, washed with ice-cold phosphate-buffered saline (PBS) and then lysed on ice RIPA buffer with protease and phosphatase inhibitors. Equal amount of protein per lane was separated by electrophoresis using 10\% Tris-Glycine polyacrylamide gels. Proteins were transferred to PVDF membranes and blocked for $1 \mathrm{~h}$ with $5 \%$ non-fat dry milk at room temperature. Overnight incubation at $4{ }^{\circ} \mathrm{C}$ with primary antibody was performed in recommended dilutions. Anti-Vinculin antibody at a dilution of 1:10,000 is used as a loading control.

\section{Transfection with siRNA}

COV434 and KGN cells were seeded in 6-well plates and cultured until $70 \%$ confluency was obtained. TransITX2 Dynamic Delivery System was used to perform siRNA transfection at concentrations of $12.5,25,50$ and $100 \mathrm{nM}$. The cells were incubated for $48 \mathrm{~h}$ post transfection, then harvested to determine the transfection efficiency by JNK expression on western blot.

\section{Real-time monitoring of cell proliferation via xCELLigence System}

The xCELLigence system uses interdigitated gold microelectrodes containing plates (E-plate) to noninvasively monitor the viability of cells using electrical impedance as the readout and generates real-time curves of cell viability and proliferation. The electronic readout of cell-sensor impedance is displayed in real-time as cell index (CI), a value directly influenced by cell attachment, spreading and/or cell proliferation. The cells were seeded in 16-well E-plate at the density of 10,000 cells per well in a final volume of $200 \mu \mathrm{L}$ and incubated at $37^{\circ} \mathrm{C}$ with $5 \%$ $\mathrm{CO}_{2}$ and continuously monitored on the RTCA system at 30 min time intervals. When the cells reached the log growth phase, they were treated with 12.5, 25, 50 and 100 $\mu \mathrm{M}$ concentrations of both SP600125 and AS601245. The effects of these inhibitors on viability and proliferation of COV434 were monitored up to $120 \mathrm{~h}$. The results were expressed by normalized CI which are derived from the ratio of CIs before and after the addition of the compounds. The normalization of $\mathrm{CI}$ arbitrarily sets $\mathrm{CI}$ to 1 at the indicated time points. Recording of CI and normalization CI was performed using the RTCA Software 1.2.

\section{Immunofluorescent staining}

For immunofluorescence studies, cells grown on coverslips were fixed with $4 \%$ paraformaldehyde for $20 \mathrm{~min}$ at room temperature, permeabilized with $0.1 \%$ Triton X-100 and then treated with superblock for $10 \mathrm{~min}$. After rinsing with DPBS-T (0.1\% Tween-20 in Dulbecco's PBS), they were incubated with pHH3 antibody in superblock at 1:50 dilution overnight at $4{ }^{\circ} \mathrm{C}$. The cells were washed with DPBS-T and incubated with fluorochrome-conjugated secondary antibody diluted in PBS for $90 \mathrm{~min}$ at $37^{\circ} \mathrm{C}$. This step was followed by rinsing the coverslips and adding Hoechst 33342 for DNA staining. The images were taken under appropriate channels using Immunofluorescence (IF) microscope. The percentage of the pHH3-positive cells was calculated after counting 500 cells at four different high-magnification areas.

\section{EdU assay}

Cells plated on coverslips were synchronized with serum starvation for $24 \mathrm{~h}$, then treated with complete media containing $2 \mu \mathrm{g} / \mathrm{mL}$ aphidicolin for $18 \mathrm{~h}$. Then, the cells synchronized at G1/S were treated with JNK inhibitor SP600125 for $1 \mathrm{~h}$ while control group was incubated in complete media. After treatments, to detect newly synthesized DNA, the cells were incubated with of $10 \mu \mathrm{M}$ EdU in culture medium for $2 \mathrm{~h}$ and fixed for imaging.

\section{Assessment of cell viability with YO-PRO ${ }^{\circledR}-1$ staining}

During apoptosis, the cytoplasmic membrane becomes slightly permeant to certain dyes, such as the green fluorescent $\mathrm{YO}_{-} \mathrm{PRO}^{{ }^{-}}-1$. After staining with $\mathrm{YO}_{-} \mathrm{PRO}^{{ }^{-}}-1$, apoptotic cells show green fluorescence while live cells show little or no fluorescence. The cells were treated with SP600125 and AS601245 for 24 h, then stained with YO$\mathrm{PRO}^{\circledR}-1(1 \mu \mathrm{M})$ and incubated for $20 \mathrm{~min}$ at $37^{\circ} \mathrm{C}$. The images were taken under appropriate channels using IF microscope. In all, 500 cells were counted at four different high-magnification areas and the percentage of the YO$\mathrm{PRO}^{\circledR}$-1-positive cells was calculated.

\section{Cell synchronization and flow cytometry analysis}

For synchronization at G1/S, cells were serum starved for $24 \mathrm{~h}$ and then treated with complete media containing $2 \mu \mathrm{g} / \mathrm{mL}$ aphidicolin for $18 \mathrm{~h}$. Then, the cells synchronized at $\mathrm{G1} / \mathrm{S}$ by aphidicolin were incubated in complete media for $12 \mathrm{~h}$. At $12 \mathrm{~h}$ after aphidicolin removal, all of the cells reached to G2/M transition $(0 \mathrm{~h})$ and at that time a set of plates were treated with JNK inhibitor SP600125 at 25 and $50 \mu \mathrm{M}$ concentrations and collected after 4,8 and $12 \mathrm{~h}$. 
For flow cytometer analysis, cells were trypsinized, washed and fixed with $70 \%$ ethanol overnight at $4{ }^{\circ} \mathrm{C}$ at indicated time points. Washed cells were treated with 50 $\mu \mathrm{g} / \mathrm{mL}$ RNase-A and then stained with $50 \mu \mathrm{g} / \mathrm{mL}$ propidium iodide. Flow cytometry analyses were performed using FACS Calibur machine and the FlowJo program. Western blot protocol described before was followed for detection of cell cycle-specific proteins.

\section{Hormone assays}

After treatment with JNK inhibitors, supernatants were collected and stored in $-30^{\circ} \mathrm{C}$ until hormone assays were performed. Electrochemiluminescence immunoassay (ECLIA) kits specific for estradiol (Elecsys ${ }^{\circledR}$ Estradiol II, Cobas) and ACTIVE $^{\circledR}$ Mullerian Inhibiting Substance/ Anti-Mullerian Hormone (MIS/AMH) enzyme-linked immunosorbent (ELISA) (Diagnostic Systems Laboratories, Inc.) for AMH were used according to the manufacturer's instructions to measure the corresponding hormone levels in the culture media. The analytical sensitivity of AMH and estradiol assays was $0.006 \mathrm{ng} / \mathrm{mL}$ and $5,0 \mathrm{pg} / \mathrm{mL}$, respectively. All analyses were performed on Cobas $^{\circledR} 6000$ analyzer series (Roche Diagnostics, USA).

\section{Statistical analysis}

Hormone levels and cell index readouts of xCELLigence system are continuous data and expressed as the mean + $\mathrm{SD}$. Analysis of variance and multiple comparison post hoc test were applied to compare the data among the groups. Statistical analyses were done using SPSS for windows 20.0 statistical package program. The percentages of viable and apoptotic cells were compared between the groups using Fisher' exact test. A $P$-value of $<0.05$ was considered significant for all statistical tests.

\section{Acknowledgements}

The authors gratefully acknowledge use of the services and facilities of the Koç University Research Center for Translational Medicine (KUTTAM), equally funded by the Republic of Turkey Ministry of Development Research Infrastructure Support Program. Findings, opinions or points of view expressed on this article do not necessarily represent the official position or policies of the Ministry of Development.

\begin{abstract}
Author details
${ }^{1}$ Graduate School of Health Sciences and School of Medicines, Koc University, Istanbul, Turkey. ${ }^{2}$ Department of Histology and Embryology, School of Medicine, Koc University, Istanbul, Turkey. ${ }^{3}$ Department of Pathology, School of Medicine, Acibadem University, Istanbul, Turkey. ${ }^{4}$ American Hospital Clinical Biochemistry Laboratories, Istanbul, Turkey. ${ }^{5}$ Department of Obstetrics and Gynecology, Gynecologic Oncology Division, School of Medicine, Koc University, Istanbul, Turkey. 'Women's Health Center, American Hospital, Istanbul, Turkey. ${ }^{7}$ Department of Obstetrics and Gynecology, The Division of Reproductive Endocrinology and Infertility, Translational Research Laboratories, School of Medicine, Koc University, Istanbul, Turkey
\end{abstract}

\section{Conflict of interest}

The authors declare that they have no conflict of interest.

\section{Publisher's note}

Springer Nature remains neutral with regard to jurisdictional claims in published maps and institutional affiliations.

Supplementary Information accompanies this paper at https://doi.org/ 10.1038/s41419-018-0459-3.

Received: 11 July 2017 Revised: 2 March 2018 Accepted: 5 March 2018 Published online: 16 March 2018

\section{References}

1. Schumer, S. T. \& Cannistra, S. A. Granulosa cell tumor of the ovary. J. Clin. Oncol. 21, 1180-1189 (2003)

2. Young, R. H., Dickersin, G. R. \& Scully, R. E. Juvenile granulosa-cell tumor of the ovary - a clinicopathological analysis of 125 cases. Am. J. Surg. Pathol. 8, 575-596 (1984).

3. Shah, S. P. et al. Mutation of FOXL2 in granulosa-cell tumors of the ovary. $N$. Engl. J. Med. 360, 2719-2729 (2009).

4. Deshpande, D. A. et al. Exploiting functional domains of GRK2/3 to alter the competitive balance of pro- and anticontractile signaling in airway smooth muscle. FASEB J. 28, 956-965 (2014).

5. Nonis, D., McTavish, K. J. \& Shimasaki, S. Essential but differential role of FOXL2Wt and FOXL2C134W in GDF-9 stimulation of follistatin transcription in co-operation with Smad3 in the human granulosa cell line COV434. Mol. Cell. Endocrinol. 372, 42-48 (2013).

6. Anttonen, M. et al. FOXL2, GATA4, and SMAD3 co-operatively modulate gene expression, cell viability and apoptosis in ovarian granulosa cell tumor cells. Plos One 9, e85545 (2014).

7. Vassal, G. et al. Juvenile granulosa cell tumor of the ovary in children: a clinical study of 15 cases. J. Clin. Oncol. 6, 990-995 (1988).

8. Wu, H. et al. Juvenile granulosa cell tumor of the ovary: a clinicopathologic study. J. Pediatr. Adolesc. Gynecol. 30, 138-143 (2017).

9. Auguste, A. et al. Molecular analyses of juvenile granulosa cell tumors bearing AKT1 mutations provide insights into tumor biology and therapeutic leads. Hum. Mol. Genet. 24, 6687-6698 (2015).

10. Kalfa, $\mathrm{N}$. et al. Extinction of FOXL2 expression in aggressive ovarian granulosa cell tumors in children. Fertil. Steril. 87, 896-901 (2007).

11. Kalfa, N. et al. Activating mutations of the stimulatory $g$ protein in juvenile ovarian granulosa cell tumors: a new prognostic factor? J. Clin. Endocrinol. Metab. 91, 1842-1847 (2006).

12. Kaye, S. B. \& Davies, E. Cyclophosphamide, adriamycin, and cisplatinum for the treatment of advanced granulosa-cell tumor, using serum estradiol as a tumor-marker. Gynecol. Oncol. 24, 261-264 (1986).

13. Rey, R. A. et al. Antimullerian hormone as a serum marker of granulosa cell tumors of the ovary: Comparative study with serum alpha-inhibin and estradiol. Am. J. Obstet. Gynecol. 174, 958-965 (1996).

14. Johnson, G. L. \& Lapadat, R. Mitogen-activated protein kinase pathways mediated by ERK, JNK, and p38 protein kinases. Science 298, 1911-1912 (2002).

15. Weston, C. R. \& Davis, R. J. The JNK signal transduction pathway. Curr. Opin. Cell Biol. 19, 142-149 (2007).

16. Johnson, R. S., Vanlingen, B., Papaioannou, V. E. \& Spiegelman, B. M. A null mutation at the c-Jun locus causes embryonic lethality and retarded cellgrowth in culture. Genes Dev. 7, 1309-1317 (1993).

17. Oktem, O., Buyuk, E. \& Oktay, K. Preantral follicle growth is regulated by c-JunN-terminal kinase (JNK) pathway. Reprod. Sci. 18, 269-276 (2011).

18. Oktem, O. \& Oktay, K. Follicle stimulating hormone regulates granulosa cell mitosis through c-Jun $n$ terminal kinase (JNK) pathway. Reprod. Sci. 15, 96a-96a (2008).

19. Stein, L. S., Stoica, G., Tilley, R. \& Burghardt, R. C. Rat ovarian granulosa cell culture: a model system for the study of cell-cell communication during multistep transformation. Cancer Res. 51, 696-706 (1991).

20. Yuksel, A. et al. The magnitude of gonadotoxicity of chemotherapy drugs on ovarian follicles and granulosa cells varies depending upon the category of the drugs and the type of granulosa cells. Hum. Reprod. 30, 2926-2935 (2015).

21. Mingo-Sion, A. M., Marietta, P. M., Koller, E., Wolf, D. M. \& Van Den Berg, C. L. Inhibition of JNK reduces G2/M transit independent of p53, leading to 
endoreduplication, decreased proliferation, and apoptosis in breast cancer cells. Oncogene 23, 596-604 (2004).

22. Miyamoto-Yamasaki, Y., Yamasaki, M., Tachibana, H. \& Yamada, K. Induction of endoreduplication by a JNK inhibitor SP600125 in human lung carcinoma A 549 cells. Cell Biol. Int. 31, 1501-1506 (2007).

23. Mili, D., Abid, K., Rjiba, I. \& Kenani, A. Effect of SP600125 on the mitotic spindle in HeLa cells, leading to mitotic arrest, endoreduplication and apoptosis. Mol. Cytogenet. 9, 86 (2016).

24. Grassi, E. S. et al. SP600125 has a remarkable anticancer potential against undifferentiated thyroid cancer through selective action on ROCK and p53 pathways. Oncotarget 6, 36383-36399 (2015).

25. Oktem, O. \& Urman, B. Understanding follicle growth in vivo. Hum. Reprod. 25, 2944-2954 (2010)

26. Moumne, L. et al. The mutations and potential targets of the forkhead transcription factor FOXL2. Mol. Cell Endocrinol. 282, 2-11 (2008).

27. Crisponi, L. et al. The putative forkhead transcription factor FOXL2 is mutated in blepharophimosis/ptosis/epicanthus inversus syndrome. Nat. Genet. 27, 159-166 (2001).

28. Schmidt, D. et al. The murine winged-helix transcription factor Fox/2 is required for granulosa cell differentiation and ovary maintenance. Development 131, 933-942 (2004).

29. Kobel, M., Gilks, C. B. \& Huntsman, D. G. Adult-type granulosa cell tumors and FOXL2 mutation. Cancer Res. 69, 9160-9162 (2009).

30. Benayoun, B. A. et al. Transcription factor FOXL2 protects granulosa cells from stress and delays cell cycle: role of its regulation by the SIRT1 deacetylase. Hum. Mol. Genet. 20, 1673-1686 (2011).

31. Fleming, N. I. et al. Aromatase is a direct target of FOXL2: C134W in granulosa cell tumors via a single highly conserved binding site in the ovarian specific promoter. PLoS One 5, e14389 (2010).

32. L'Hote, D. et al. Discovery of novel protein partners of the transcription factor FOXL2 provides insights into its physiopathological roles. Hum. Mol. Genet. 21, 3264-3274 (2012).
33. Kim, J. H. et al. Differential apoptotic activities of wild-type FOXL2 and the adult-type granulosa cell tumor-associated mutant FOXL2 (C134W). Oncogene 30, 1653-1663 (2011).

34. Liu, Z. et al. FOXO1/3 and PTEN depletion in granulosa cells promotes ovarian granulosa cell tumor development. Mol. Endocrinol. 29, 1006-1024 (2015).

35. Richards, J. S. et al. Either Kras activation or Pten loss similarly enhance the dominant-stable CTNNB1-induced genetic program to promote granulosa cell tumor development in the ovary and testis. Oncogene 31, 1504-1520 (2012).

36. Kinross, K. M. et al. An activating Pik3ca mutation coupled with Pten loss is sufficient to initiate ovarian tumorigenesis in mice. J. Clin. Invest. 122, 553-557 (2012).

37. Bittinger, S., Alexiadis, M. \& Fuller, P. J. Expression status and mutational analysis of the PTEN and P13K subunit genes in ovarian granulosa cell tumors. Int. J. Gynecol. Cancer 19, 339-342 (2009).

38. Rico, C. et al. Pharmacological targeting of mammalian target of rapamycin inhibits ovarian granulosa cell tumor growth. Carcinogenesis 33, 2283-2292 (2012).

39. Zhang, $\mathrm{H}$. et al. Characterization of an immortalized human granulosa cell line (COV434). Mol. Hum. Reprod. 6, 146-153 (2000).

40. Nishi, Y. et al. Establishment and characterization of a steroidogenic human granulosa-like tumor cell line, KGN, that expresses functional folliclestimulating hormone receptor. Endocrinology 142, 437-445 (2001).

41. Bayasula et al. Establishment of a human nonluteinized granulosa cell line that transitions from the gonadotropin-independent to the gonadotropindependent status. Endocrinology 153, 2851-2860 (2012).

42. Oktem, O., Senbabaoglu, F., Muftuoglu, M. \& Urman, B. Real-time analysis of the growth of human granulosa cells using an impedance-based signal processing system: a new technology for translational research in human reproduction. Hum. Reprod. 28, i52-i55 (2013).

43. Oktem, O., Buyuk, E. \& Oktay, K. Preantral follicle growth is regulated by c-JunN-terminal kinase (JNK) pathway. Reprod. Sci. 18, 269-276 (2011). 